\title{
Determination of the magnetic loop configuration of a solar flare
}

\author{
Y. Hanaoka and T. Sakurai \\ National Astronomical Observatory of Japan, 2-21-1 Osawa, Mitaka, Tokyo 181-8588, Japan
}

(Received June 13, 2000; Revised September 29, 2000; Accepted October 10, 2000)

\begin{abstract}
Observationally derived three-dimensional magnetic loop structures of solar flares tell us what type of magnetic configurations is actually responsible for the reconnection/energy release in solar flares. So far, the loop configuration has been discussed on a rather schematic basis, because of insufficient data coverage. However, now we have data obtained with various advanced instruments, which are essentially important to define the three-dimensional magnetic configuration of flare loops. In this paper, we briefly present how the loop configuration of the solar flares can be determined based on recent advanced observations. We focus on the flares caused by emerging flux activity in region NOAA 8100, which appeared in October-November 1997. We present here the presumed quadruple structure of the magnetic loops of the analyzed flares in three dimensions, and suggest that the emergence of small loops can give rise to large-scale phenomena like jets and CMEs.
\end{abstract}

\section{Introduction}

Energy release in solar flares is presumed to be caused by magnetic reconnection between coronal loops. Observations of the solar flares give the magnetic configuration which is actually responsible for the reconnection.

Collisions between a magnetic loop emerging from below the photosphere and a pre-existing overlying magnetic loop are a typical cause of magnetic reconnections in solar flares. Such a reconnection process has been theoretically discussed in two dimensions, as a collision of anti-parallel magnetic fields by many authors (e.g. Heyvaerts et al., 1977; Yokoyama and Shibata, 1995). However, the actual configuration of the loops in three dimensions should be clarified on the basis of observations. Using various types of data sets, many authors have attempted to derive the threedimensional configuration of the interacting loops and inferred quadrupolar structures of the reconnecting loops (e.g. Machado et al., 1983; Hanaoka, 1997; Aschwanden et al., 1999). In many cases, it was found that the reconnection in such flares occurs between non anti-parallel magnetic fields. However, due to insufficient data coverage or insufficient spatial resolution, the loop configuration has been discussed so far on a rather schematic basis. The progress of recent observations enables us to figure out more realistic threedimensional quadrupolar loop configurations. In this paper, we present how the configuration of the flare loops is derived based on the advanced observations.

We analyzed flares in an active region NOAA 8100, which are caused by emerging flux activity. The active region appeared in October-November 1997, and the activity of this region was observed by various advanced instruments as follows.

Copy right (C) The Society of Geomagnetism and Earth, Planetary and Space Sciences (SGEPSS); The Seismological Society of Japan; The Volcanological Society of Japan; The Geodetic Society of Japan; The Japanese Society for Planetary Sciences.

\section{(1) Photospheric magnetograms}

Longitudinal magnetograms were taken with the $\mathrm{SOHO} /$ MDI. Although its spatial resolution is moderate, its continuous time-coverage is essentially important to study the evolution of the magnetic field, which is responsible for the energy build-up and eventually causes a flare. The photospheric magnetogram data can be extrapolated to coronal magnetic field lines.

(2) Images of the corona

Coronal loop structures are seen in the images taken with the Yohkoh/SXT and the SOHO/EIT. The loop structure can be compared with the extrapolated magnetic field lines based on the photospheric magnetograms.

(3) X-ray, radio, and $\mathrm{H} \alpha$ images of the solar flares

These data reflect various aspects of the energy release due to solar flares. High-resolution pictures are useful to identify the footpoints of the flare loops.

In the subsequent sections, we will present our study of the three-dimensional configuration and evolution of the reconnecting loops, based on the analysis of these data.

\section{Evolution of Region NOAA 8100}

Figure 1(a) shows the variation of the total magnetic flux of region 8100 measured on the MDI magnetograms. The inset picture in Fig. 1(a) is a sample white light image of region 8100. As shown in Fig. 1(a), this region showed a rapid development over a week, and caused many flares and CMEs. Besides the development of the region itself, we can find the growths of some emerging flux regions. In this paper, we concentrate our study on the emerging flux denoted by arrows in Figs. 1(c)-(f). The development of a positive parasitic polarity region is clearly seen, and this is a part of an emerging flux region. We caluculated its magnetic flux, integrating the positive polarity flux in the about $20^{\prime \prime} \times 20^{\prime \prime}$ region including the parasitic polarity. The increase of its magnetic flux is shown in Fig. 1(b). This emerging flux re- 

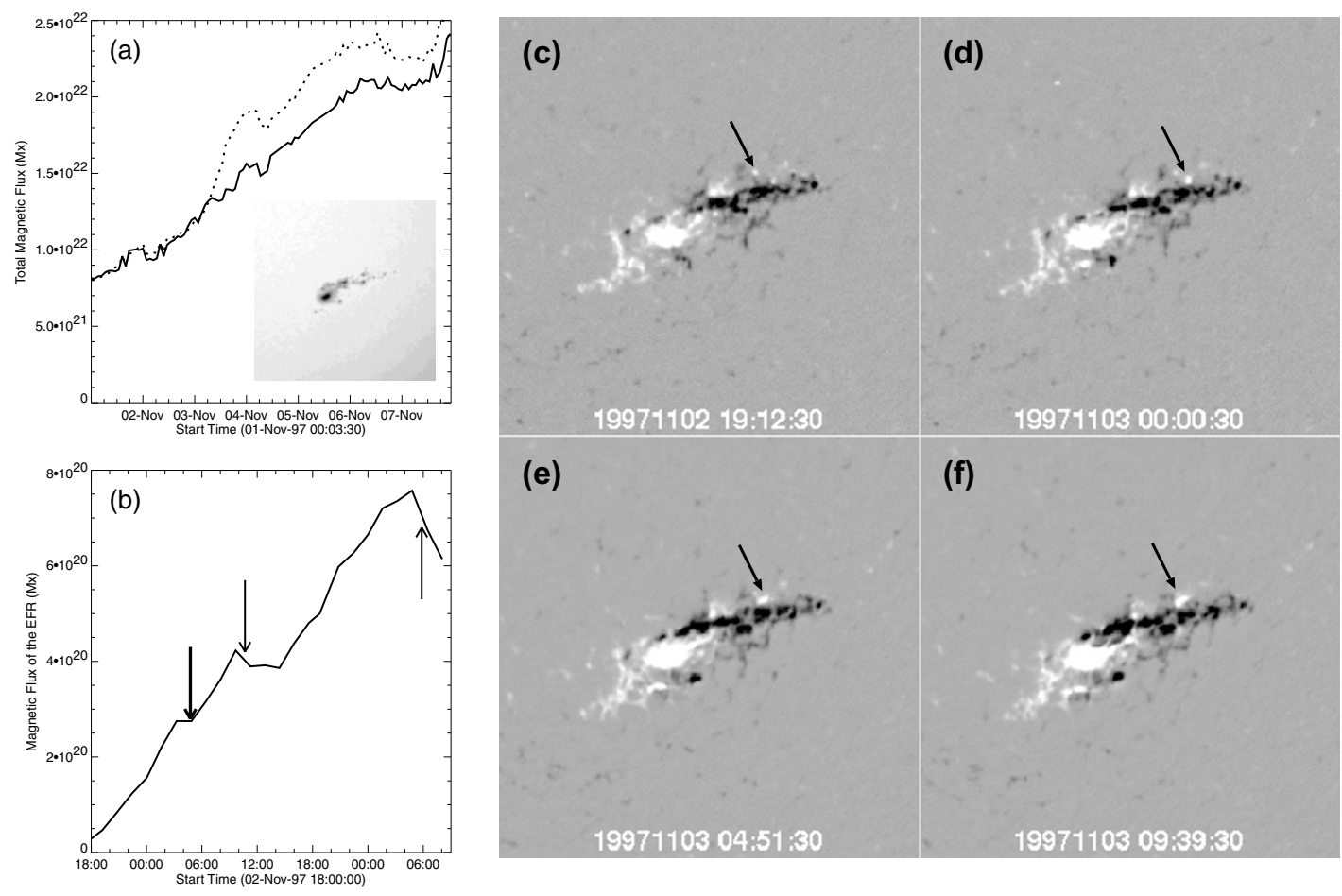

(e)

(f)

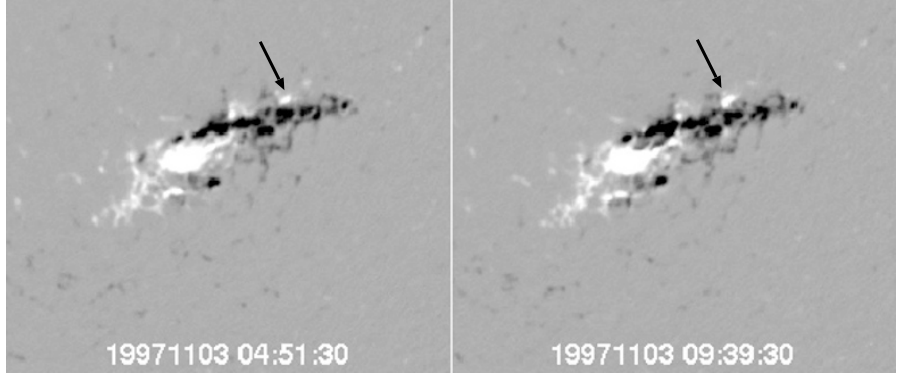

Fig. 1. (a) Total magnetic flux variation of NOAA 8100 measured on the SOHO/MDI magnetograms. The solid line shows the total flux of the positive polarity, and the dashed line shows that of the negative polarity. The inset is a white light picture of NOAA 8100 taken with the MDI. (b) Magnetic flux variation of the positive polarity component of an emerging flux region shown in (c)-(f). The arrows show homologous flares caused by the emerging flux region. The first one, shown with a thick arrow, is particularly discussed in Section 3. (c)-(f) Magnetograms of NOAA 8100 taken with the MDI. White and black stand for the positive and negative polarities, respectively. Arrows show an emerging flux region and its growth.

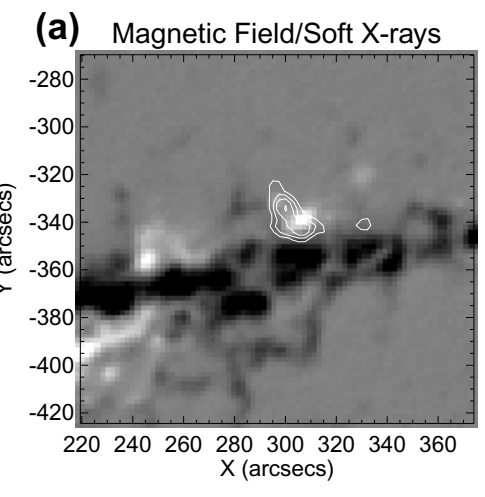

(c) MDI Magnetic Field $/ 17 \mathrm{GHz}$

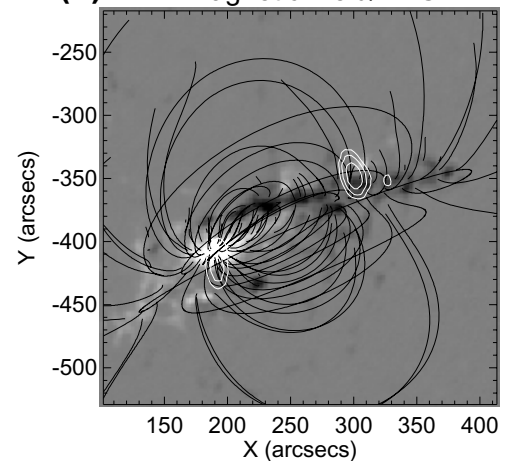

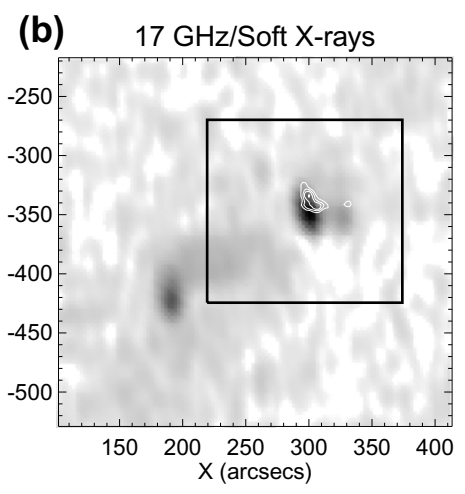

(d)

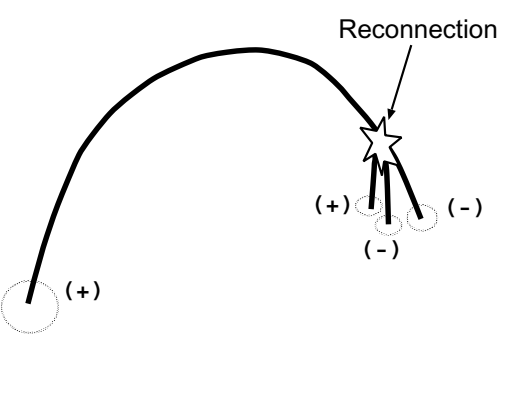

Fig. 2. (a) Photospheric magnetogram taken with the SOHO/MDI at 04:51 UT on November 3 overlaid with the contours of a soft X-ray picture of a C8.6 flare at 04:38 UT taken with the SXT. North is to the top. (b) Radio picture of the flare at 04:34 UT taken with the Nobeyama Radioheliograph. Contours of the soft X-ray image are overlaid. The field of view of panel (a) is shown by a square. (c) Magnetogram taken with the SOHO/MDI overlaid by the extrapolated potential magnetic field lines and the contours of the radio map. (d) Presumed quadruple configuration of the magnetic loops, which causes the flare. 

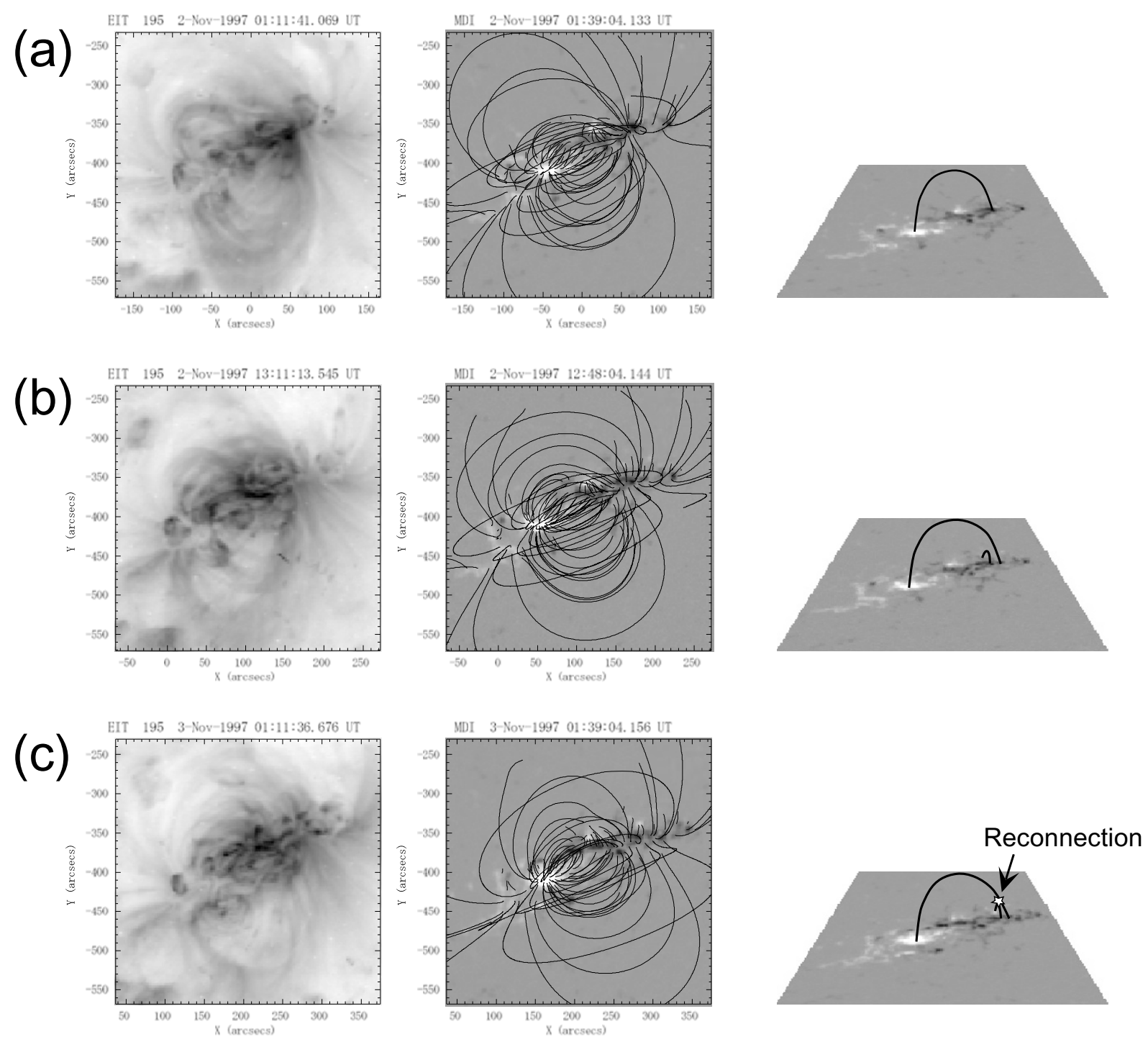

Fig. 3. Evolution of the coronal loops and the magnetic field in region 8100. (From left to right) Fe XII $195 \AA$ Amages taken with the SOHO/EIT; photospheric magnetograms taken with the SOHO/MDI, overlaid with the potential magnetic field lines; schematic drawings of the emerging loop and the overlying loop, which cause the flare, on the magnetograms.

gion appeared on November 2, and showed a rapid growth for about 48 hours. During its growth, it caused numerous active phenomena. Particularly, three flares denoted by arrows in Fig. 1(b) showed a homologous nature. The appearance of these flares were basically a compact brightening. However, all of these flares were accompanied by a jet-like ejection, which originated in the emerging flux region. Furthermore, all of these flares caused CMEs. Images of the corona in the Fe XII line taken with the SOHO/EIT after the flares/CMEs show a coronal dimming due to the CMEs just above the emerging flux region. Therefore, these observations suggest that a local, small scale reconnection between an emerging loop and an overlying loop causes a large scale restructuring of the magnetic field such as a jet and a CME. Delannée and Aulanier (1999) suggested that the CME source region included NOAA 8102 in the northern hemisphere as well as NOAA 8100 in the southern hemisphere. Their result implies that the activity of the emerging flux causes the disruption of trans-equatorial coronal loops.
However, in this paper, we only describe the interaction between the emerging loop and the overlying loop, and the discussion about the relation between the emerging flux and the CMEs will appear in a forthcoming paper.

Since a C8.6 flare at 04:38 UT on November 3 (shown with a thick arrow in Fig. 1(b)) is a typical example of the flares and it was well observed by various instruments, we present a detailed analysis of this particular flare in the next section.

\section{Magnetic Configuration of the Flares}

Figure 2(a) shows an overlay of a soft X-ray image in contours of the C8.6 flare at 04:38 UT on November 3 taken with the Yohkoh/SXT on an MDI magnetogram. The brightest, elongated soft X-ray source is found above a parasitic positive polarity region, which is the one denoted by arrows in Figs. 1(c)-(f). Therefore, the source is located at the emerging flux region, and this source corresponds to the emerging loop, which caused flaring. A hard X-ray source 
shows a structure similar to the soft X-ray source (not shown in Fig. 2). The X-ray images give an impression that this flare is rather a compact, confined one, but a radio picture shown in Fig. 2(b) shows a remote bright point as well as a source located at the soft X-ray source region. An overlay of a contour map of the radio sources on a magnetogram (Fig. 2(c)) shows that the remote radio source is located at the major following polarity of the active region at the coordinate [190, -420], and the magnetic field lines based on the potential field extrapolation (Sakurai, 1982) shown in Fig. 2(c) indicate a connection between the two radio sources. A small loop at the emerging flux region, which corresponds to the emerging loop, can be also found in Fig. 2(c). Therefore, this flare is presumed to be caused by a collision between the emerging flux and the overlying large loop, and the three-dimensional structure of the loops can be determined from the above results as shown in Fig. 2(d).

\section{Evolution of the Magnetic Field}

As shown in the previous section, we can specify the footpoints of the flare loops based on the X-ray/radio observations of the flares, and also we can specify the structure of the flare loops based on the magnetograms and the magnetic field extrapolation. In this section, the evolution of the magnetic field, which eventually produced such a loop structure and caused the flares, is described. Figure 3 shows coronal images, magnetograms and extrapolated potential field lines, and schematic drawings of the flaring loops. Note that the potential field lines are extrapolated on the basis of the spherical boundary condition, even though the magnetograms in the schematic drawings are shown on a plane.

Before the appearance of the emerging flux region, there is a connection between the major preceding and the major following polarity regions as shown in Fig. 3(a). The structure of the large scale loops seen in a coronal image taken by the EIT is not much different from the extrapolated potential field. In Fig. 3(b), the emerging flux loop appeared.
It grew and eventually collided with the pre-existing large loop as shown in Fig. 3(c), and this collision caused a flare. The positions of the footpoints of the flaring loops are identified based on Fig. 2, and the shape of the loops can be known from the extrapolated magnetic field lines. During the growth of the emerging loop, it caused flares and CMEs recurrently, which showed a homologous nature.

In this paper, we have just shown an example of results from the study of the configuration of the flare loops and their evolution. A more thorough study on the observationally-determined three-dimensional configuration of flares is under way, and will give a definite contribution to the understanding of the physics of magnetic reconnection.

This work is a part of the results obtained at the CDAW (coordinated data analysis workshop) held at NASA/GSFC in April, 1999. We are grateful to the organizers of the workshop, the SOHO science team who provided the data obtained by SOHO, and those who preprocessed the data for the workshop.

\section{References}

Aschwanden, M. J., T. Kosugi, Y. Hanaoka, M. Nishio, and D. B. Melrose, Quadrupolar magnetic reconnection in solar flares. I. Three-dimensional geometry inferred from Yohkoh observations, Astrophysical J., 526, 1026-1045, 1999.

Delannée, C. and G. Aulanier, CME associated with transequatorial loops and a bald patch flare, Solar Physics, 190, 107-129, 1999.

Hanaoka, Y., Double-loop configuration of solar flares, Solar Physics, 173, 319-346, 1997.

Heyvaerts, J., E. R. Priest, and D. M. Rust, An emerging flux model for the solar flare phenomenon, Astrophysical J., 216, 123-137, 1977.

Machado, M. E., B. V. Somov, M. G. Rovira, and C. de Jager, The flares of April 1980 - A case for flares caused by interacting field structures, Solar Physics, 85, 157-184, 1983.

Sakurai, T., Green's function methods for potential magnetic fields, Solar Physics, 76, 301-321, 1982.

Yokoyama, T. and K. Shibata, Magnetic reconnection as the origin of X-ray jets and H-alpha surges on the Sun, Nature, 375, 42-44, 1995.

Y. Hanaoka (e-mail: hanaoka@solar.mtk.nao.ac.jp) and T. Sakurai 